\title{
ANTIOXIDANT EFFECT OF VITAMINS COMBINED A, C, AND E DOSE ON TYPE 2 DIABETIC PATIENTS
}

\author{
GOZIF MOHAMMED N OMAR ${ }^{1 *}$, FADEL YOUSIF AL-ARABI², MANSOUR ABDULNABI H MEHDI², \\ MADHUKAR M FAWADE ${ }^{1}$
}

\begin{abstract}
${ }^{1}$ Department of Biochemistry, Dr. Babasaheb Ambedkar Marathwada University, Aurangabad - 431 004, India. ${ }^{2}$ Dr. Rafiq Zakaria College for Woman, Dr. Babasaheb Ambedkar Marathwada University, Aurangabad - 431 001, India. Email: Gozifalamiriomar@gmail.com
\end{abstract}

Received: 4 January 2019, Revised and Accepted: 30 January 2019

ABSTRACT

Objectives: This study is to investigate the effect of oral Vitamins combined (A, C, and E) with metformin on the levels of fasting blood sugar (FBS), postprandial blood sugar (PPBS), hemoglobin (HbA1c), total cholesterol (TC), triglycerides (TG), high-density lipoprotein (HDL), and low-density lipoprotein (LDL) in Type 2 diabetic patients.

Methods: A total of 32 patients with Type 2 diabetes were divided randomly into A and B groups, i.e., 16 per each group. Group A received metformin only twice a day while Group B received one tablet of Antox and metformin twice a day for 3 months. FBS, PPBS, HbA1c, TC, TG, LDL, and HDL were measured before and after the dose, and the results were analyzed statistically.

Results: A significant decreases in FBS, PPBS, HbA1c, TC, TG, and LDL whereas results showed increasing significance in HDL level that seen in the patients of Group B which received Antox and metformin compared to the group which received metformin only. In conclusion, the results indicate that daily consumption of supplementary vitamins with metformin leads to improving blood glucose and lipids in patients with Type 2 diabetes and reducing the risk of complications. Hence, the dose can be used combined Vitamins A, C, and E with metformin in the treatment of Type 2 diabetes by maintaining good glycemic control.

Keywords: Type 2 diabetes, Blood sugar, Serum lipids, Vitamins A, C, and E.

(C) 2019 The Authors. Published by Innovare Academic Sciences Pvt Ltd. This is an open access article under the CC BY license (http://creativecommons. org/licenses/by/4. 0/) DOI: http://dx.doi.org/10.22159/ajpcr.2019.v12i4.31216

\section{INTRODUCTION}

Diabetes mellitus is a group of metabolic disorders characterized by hyperglycemia resulting from disability in insulin action, insulin secretion, or both [1]. Recent studies indicate that 171 million people worldwide suffer from diabetes in 2000 and the number of people with diabetes is expected to rise to 366 million by 2030 [2,3].

An antioxidant is a molecule that inhibits the oxidation of other molecules, and a substance which slows down the damage that can be caused to other substances by the effects of oxygen. Food which contains antioxidants is thought to be very good for diabetes. However, later the biologists realized the significance of antioxidants in health that was in the 1960s publications of vitamins and flavonoids [4]. Usually, there are many antioxidants including $\mathrm{A}, \mathrm{C}$, and E. Vitamin $\mathrm{C}$ is an important antioxidant in a human which is capable of scavenging oxygen-derived free radicals [5]. A previous study has shown a decrease in basal Vitamin $\mathrm{C}$ level in Type 2 diabetes mellitus [6]. Another study indicated that daily consumption supplementary Vitamin $\mathrm{C}$ can be useful in reducing lipids and blood glucose in patients with Type 2 diabetes and therefore degrading the risk of complications [7]. Vitamins C and E are known for their beneficial effects on blood sugar, serum lipids, and glycated hemoglobin (HbA1c) [8]. In addition to its role as an antioxidant, studies have also shown the role of vitamin A in the upregulating the antioxidant enzyme functions in the body. In the study on obese mice, the results have shown that Vitamin A supplementation resulted in reducing hyperglycemia and hyperlipidemia [9].

This study was conducted to evaluate and to measure the effect of Vitamins A, C, and E supplemented along with metformin on levels of fasting blood sugar (FBS), postprandial blood sugar (PPBS), total cholesterol (TC), low- and high-density lipoprotein (LDL and HDL), triglycerides (TG), and levels of HbA1c in patients with Type 2 diabetes.

\section{MATERIALS AND METHODS}

A total of 32 patients with Type 2 diabetes mellitus participated, in the study which has continued for 3 months and the study was performed in accordance with the principles outlined in the Declaration of Helsinki has been approved by Dr. Babasaheb Ambedkar Marathwada University, Aurangabad, India, and the Ethics Committee at Halmeen Hospital, and AL-Amal Medical Diagnostic center Lahj, Yemen. All patients give their informed consent before their inclusion in the study. These are patients diagnosed with Type 2 diabetes of age group between 30 and 70 years who were dependent on metformin dose so that the treatment was used by having Glucovance, each tablet of Glucovance $500 \mathrm{mg} / 2.5 \mathrm{mg}$, contains $500 \mathrm{mg}$ Metformin and $2.5 \mathrm{mg}$ of glibenclamide, manufacturer Merck Sante s.a.s. 2, rue du pressoir Vert 45400 SEMOY- FRANCE. Patients who have fasting blood glucose level $>300 \mathrm{mg} / \mathrm{dl}$, medical illnesses including metabolic, type 1 diabetes, pregnancy, and age $<30$ years, and age $>70$ years were excluded from the study. None of the subjects was smoker, drinker or had been taking any psychotropic drug. Patients who have received Vitamins A, C, and E or any other antioxidant over the past 3 months were also excluded from the study. The period of the present study was from September 2016 to February 2017.

Patients with Type 2 diabetes were divided randomly into two groups: A and B of 16 patients in each group. The sample size was calculated with the standard deviation taken from a study and the level of significance at $\leq 0.05$. The drug was given to patients for the duration of 1 month at a time. Patients were required to return the unused drugs and container during the follow-up treatment, so $95 \%$ consumption was considered to be suitable. Drugs were decoded at the end of a trial. Group B received Antox dose a dietary supplement produced by Arab company for pharmaceuticals and plant - Egypt with Metformin and Group A received metformin only. The dose Antox content on combined 
with Vitamins A, C, and E was given twice a day to Group B. At the same time, all patients received tablet metformin $500 \mathrm{mg}$ twice daily orally with breakfast and dinner; similar, doses were kept throughout the study. If we found increased FBS level $300 \mathrm{mg} / \mathrm{dl}$ through the study time, then we excluded that patient from the study. No disease condition or infection happened to these study patients through the study period.

After the study performance was over, the subjects were given over to the specialist physician of diet; all patients were maintained on their normal dietary type while reducing their consumption of Vitamins A, C, and E rich food during the study. A patient was commanded by a physician to use a basic food list that contained food suitable for diabetic patients. Investigations of values were considered normal; FBS $<126 \mathrm{mg} / \mathrm{dl}, \mathrm{TC}<200 \mathrm{mg} / \mathrm{dl}, \mathrm{TG}<160 \mathrm{mg} / \mathrm{dl}$, HDL-cholesterol 35-55 mg/dl, LDL- cholesterol $<100 \mathrm{mg} / \mathrm{dl}$, and HbA1c 6 . Age and sex were examined and recorded. Blood samples were drawn before and after a meal from the patients to measure levels of FBS, PPBS, TC, LDL and HDL, TG, and glycated HbA1c. These were measured before the initiation of supplementation with Vitamins A, C, and E. Which have been used for doing these investigations, the following methods were used: Kit Spinreact.

- Estimation of serum glucose by Glucose oxidase-peroxidase method.

- Estimation of serum TC by cholesterol oxidase-peroxidase method.

- Estimation of serum TG by Glycerol-3-oxidase-peroxidase method.

- Estimation of serum HDL by cholesterol oxidase method.

- Estimation of serum LDL by Friedewald formula

- Estimation of glycated HbA1c by SD A1cCare."

After 3 months of supplementation with Vitamins A, C, and E, patients were examined again, and the tests were repeated.

\section{Statistical analysis}

Results were expressed as mean \pm standard deviation. Group differences were calculated by SPSS paired t-test. Two-tailed $\mathrm{p}$ value was used throughout the analysis, and the p values which were $\leq 0.05$ were judged statistically significant ( $\mathrm{p} \leq 0.05)$. IBM SPSS statistical (version 21 ) was used.

\section{RESULTS}

The mean age of the patients with diabetes mellitus in control group was 49.06+10.70 years and experimental group 48.68+11.47 years; it was not significantly different from each other (Table 1). Fasting, postprandial blood glucose, HbA1c, TC, TG, HDL, and LDL did not differ among subjects before treatment in the control group and experimental group $(\mathrm{p} \leq 0.05)$

In the control group, levels of FBS reduced significantly $(p \leq 0.05)$. PPBS also reduced but did not reach the level of statistical significance $(\mathrm{p} \leq 0.05)$. At the same time, levels of $\mathrm{HbA1c}$ had no improvement after 3 months of treatment compared to pre-treatment levels. Increasing the level of lipid profile was seen, and it was not a statistically significant level of reduction in HDL so that there was no improvement (Table 2). In the experimental group, reduction in FBS from 192.13 to $158.94 \mathrm{p}=0.000$, PPBS from 268.50 to $231.75 \mathrm{p}=0.000, \mathrm{HbA} 1 \mathrm{c}$ from 9.48 to $8.23 \mathrm{p}=0.000$, TC from 177.56 to $165.50 \mathrm{p}=0.001$, TG from 168.56 to $157.88 \mathrm{p}=0.048$, and LDL from 104.69 to $90.56 \mathrm{p}=0.000$ were significant, and at the same time level of HDL reached to statistically significant $p=0.000$, where it increased from 39.56 to 44.06 ) (Table 3).

To test whether oral supplementation of Antox containing Vitamins A, $\mathrm{C}$, and $\mathrm{E}$ is better in reducing level fasting blood glucose, postprandial blood glucose, glycosylated hemoglobin, TC, TG, and LDL as compared to control group, in comparing the effects of medication metformin in control group and metformin with Antox in experimental group after 3 months of conducting the treatment, taking into concern the change from baseline values of these parameters. Decrease in fasting, postprandial blood glucose, and glycosylated hemoglobin level were significant after 3 months in the experimental group compared to the control group. In contrast, plasma HDL increased significantly in the experimental group compared to the control group. At the same time, lipid profile (TC, TG, and LDL) levels decreased significantly in the experimental.

In the course of the trial, no serious adverse effect was reported, and no abnormalities in laboratory test were found during the study period.

\section{DISCUSSION}

Showed present study a significant decrease in level FBG, PPBG, HbA1c, TC, TG, and LDL and an increase in HDL level the diabetes patients which reached to the significance level after the treatment by use (Antox) supplementation along with metformin in patients of type 2 diabetes. To my knowledge of experience, this is the first report related to a significant improvement in all the above parameters after concomitant use of Antox (Vitamins A, C, and E) with metformin. The results of this study are in agreement with previously published data showing improvement in glycemic control with Vitamin supplementation A or C or E or together. In this study, patients received metformin which is an established firstline drug for the treatment of Type 2 diabetes. Hence, it is difficult to say whether this beneficial effect of Antox supplement could be related to its effect on the underlying disease or correction of the inadequate Vitamins A, C, and E status, but it showed a beneficial effect in the group which used Vitamins A, C, and E (Antox) with metformin better than the second group which used only metformin. It is well known from previous studies that there is an increased production of damaging free radicals in Type 2

Table 1: Age and distribution

\begin{tabular}{llll}
\hline Variables & Age & Male & Female \\
\hline Group A & $49.06 \pm 10.70$ & 8 & 8 \\
Group B & $48.68 \pm 11.47$ & 8 & 8 \\
\hline
\end{tabular}

Values are given as Mean+SD, 32 all groups, Group A: Control group,

Group B: Experimental group

Table 2: Group A control the effect of metformin only on FBS, PPBS, HbA1c, TC, TG, HDL, and LDL in patients with type 2 diabetes after 3 months of treatment

\begin{tabular}{llll}
\hline Parameter & Before treatment & After treatment & p \\
\hline FBS & $184.06 \pm 49.03$ & $174.13 \pm 46.60$ & 0.029 \\
PPBS & $257.94 \pm 46.74$ & $248.63 \pm 49.82$ & 0.085 \\
HbA1c & $8.98 \pm 1.59$ & $8.99 \pm 1.48$ & 0.926 \\
TC & $174.38 \pm 36.91$ & $178.56 \pm 44.76$ & 0.345 \\
TG & $164.56 \pm 63.19$ & $178.25 \pm 65.42$ & 0.159 \\
HDL & $42.44 \pm 7.23$ & $40.56 \pm 9.32$ & 0.090 \\
LDL & $99.19 \pm 39.59$ & $102.13 \pm 44.59$ & 0.431 \\
\hline
\end{tabular}

$\mathrm{n}=16$ in number, FBS: Fasting blood sugar, PPBS: Postprandial blood sugar, HbA1c: Glycosylated hemoglobin, TC: Total cholesterol, TG: Triglyceride, HDL: High-density lipoprotein, LDL: Low-density lipoprotein, are measured in $\mathrm{mg} / \mathrm{dl}$

Table 3: Group B, experimental, effect of metformin with Antox (A, C, and E) on FBS, PPBS, HbA1c, TC, TG, HDL, and LDL in patients with type 2 diabetes after 3 months of treatment

\begin{tabular}{llll}
\hline Parameter & Before treatment & After treatment & p \\
\hline FBS & $192.13 \pm 38.91$ & $158.94 \pm 27.16$ & 0.000 \\
PPBS & $268.50 \pm 39.86$ & $231.75 \pm 38.55$ & 0.000 \\
HbA1c & $9.48 \pm 0.99$ & $8.23 \pm 0.97$ & 0.000 \\
TC & $177.56 \pm 49.28$ & $165.50 \pm 48.34$ & 0.001 \\
TG & $168.56 \pm 55.47$ & $157.88 \pm 50.58$ & 0.048 \\
HDL & $39.56 \pm 4.80$ & $44.06 \pm 3.82$ & 0.000 \\
LDL & $104.69 \pm 49.24$ & $90.56 \pm 49.59$ & 0.000 \\
\hline
\end{tabular}

$\mathrm{n}=16$ in number, FBS: Fasting blood sugar, PPBS: Postprandial blood sugar HbA1c: Glycosylated hemoglobin, TC: Total cholesterol, TG: Triglyceride, HDL: High-density lipoprotein, LDL: Low-density lipoprotein, are measured in $\mathrm{mg} / \mathrm{dl}$ 
diabetes patients. Glucose auto-oxidation, protein glycosylation, in a formation of the polyol pathway, and advanced glycation end products are involved in the generation of oxidative stress, which involved in the origin of both Type 1 and 2 diabetes [10]. The imbalance between the reactive types and the antioxidants leads to the production of oxidative stress which causes the development of the condition to diabetes. There are beneficial effects of antioxidant on the complications of diabetes in which reduce oxidative stress and relieve diabetes and its complications [11,12].

The protection against damage can be provided free radical-scavenging by antioxidants. The first important observation of the studies was a significant reduction in FBS, PPBS, HbA1c, TC, TG, and LDL in varying periods of time when adding Vitamins (A, C, and E) to the experimental groups either combined or single. In previous studies, combined doses (500 mg/day twice) for 4 months, of Vitamins $\mathrm{E}$ and $\mathrm{C}$ in addition to the normal food improved the plasma glucose (FBS) and lipid profile in patients with Type 2 diabetes [8]. Another study reported that the kidney function of diabetic animals can be improved. In a trial including supplementation with combined Vitamins $\mathrm{C}$ and $\mathrm{E}$ that group given the antioxidants had a significantly less concentration of urinary microalbumin secretion in comparison to the control group [12]

Supplementation of Vitamin C significantly reduced FBG and HbA1c levels in Type 2 diabetic patients. In the previous study, supplementation with $500 \mathrm{mg}$ /day Vitamin C in diabetic patients no changes in FBG and $\mathrm{HbA1c}$ in comparison with placebo this may be related to revealed a low dose of Vitamin C [13]. An earlier study confirmed on Type 2 diabetes patients, that daily consumption $1000 \mathrm{mg}$ supplementary Vitamin C can be useful in significantly reducing glucose and lipids in patients with Type 2 diabetes and hence reducing the risk of complications [7]. The study proposed that supplementation of Vitamin $\mathrm{C}$ significantly reduces FBS, PPBG, and HbA1c (good glycemic control) [14]. Another study indicating that after treatment with Vitamin $\mathrm{C}$ along with metformin compared to placebo with metformin, FBS, PPBS, and HbA1c levels showed a significant improvement after 12 weeks of treatment with Vitamin C [15]. Another study showed that there is a significant decrease in levels of serum LDL and HbA1c in the patients who received supplementation $1000 \mathrm{mg} /$ day of Vitamin C during 6 weeks [16]. In another study, an improvement appeared in a decrease of TC using $2 \mathrm{~g}$ of Vitamin C for 90 days [17].

Vitamin E improves results which are correlated with pancreas physiology in diabetes which may improve functional results of diabetes [18]. In a study carried out to evaluate the ability of oral supplementation of Vitamin E (900 mg dday) in Type 2 diabetes patients over 4 months period, it was shown that the supplementation

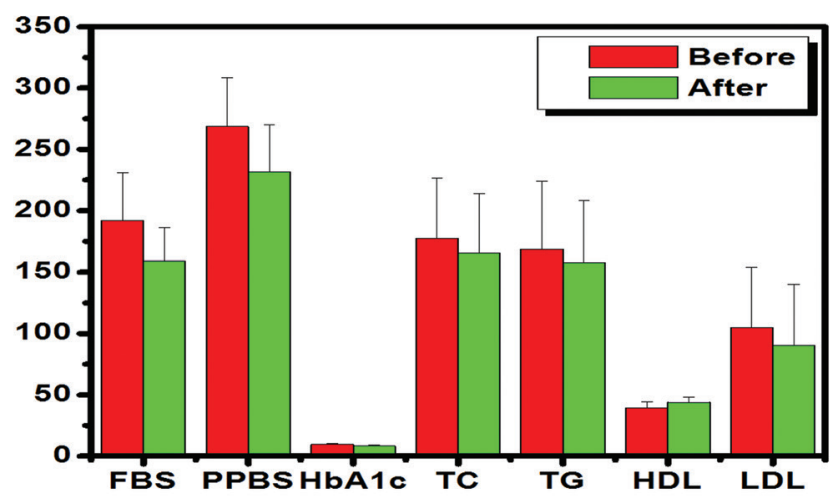

Fig. 1: Levels of decrease or increase (improvement) are shown the mean values of FBS: Fasting blood sugar, PPBS: Postprandial blood sugar, HbA1c: Glycosylated hemoglobin, TC: Total cholesterol, TG: Triglyceride, HDL: High-density lipoprotein, LDL: Low-density lipoprotein, before (red bars) and after (green bars) supplementation/treatment with doses of Antox (Vitamins A, C, and E) of Vitamin E decreased insulin resistance and improved glucose uptake [19]. As revealed in another study that supplementation of 100 IU of Vitamin E could reduce the HbA1c levels in type 1 diabetic patients [20]. Some studies have also shown that Vitamin E can be a helpful addition to decreasing oxidative stress in Type 2 diabetes. This was confirmed by a previous study in which HbA1c and other proteins were reduced with each 600 or $1200 \mathrm{mg} /$ day of Vitamin E for 2 months [21]. The previous studies have shown that Vitamin $\mathrm{E}$ is helpful in leading significantly reducing approximately $60 \%$ of plasma LDL oxidation in diabetic patients [18]. As shown in another study, it is significantly reducing cardiovascular effects in people with diabetes [22].

It has been observed that the Asian developing countries and the lower poorer groups in the United States where diabetes is on the increase have a deficient Vitamin A status [23]. An increased concentration of both cellular retinoic acid binding protein and cellular retinol binding protein in the islets results from a deficiency of Vitamin A and may reduce insulin release. Retinoic acid has been recently identified as a regenerator of rat insulinoma cell line. It is also reported to raise glucokinase activity in RINmF5 cell line as well as fetal islets and differentiated adult islets boasting of a role in the regulation of islet function and insulin release [24]. Furthermore, retinoid biology has vital importance in diabetes progression. Both too little retinol and an excess of it can have harmful effects on disease onset. A bond has been found between diabetes and deficient Vitamin A levels indicating Vitamin A supplementation have a role in Type 2 diabetes biology [25].

According to previous findings, single vitamin and a low single dose in the same time did not cause significant benefits in all cases and all parametric (glucose and lipid profile) in diabetes patients, but increased dose causes significant benefits. In the present study, combined Vitamins A, C, and E (ANTOX) twice daily for 3 months caused a significant decrease in FBG, PPBG, HbA1c, TC, TG, and LDL and an increasingly significant in HDL level after treatment by Antox supplementation along with metformin in patients of type 2 diabetes (Fig. 1).

\section{CONCLUSION}

Treatment with Antox (combined Vitamins A, C, and E) twice daily with metformin for 3 months period was well tolerated and devoid of any side effects. This treatment has not any substantial side effects. It has led to improvement in that it caused a decrease FBG, PPBG, HbA1c, TC, TG, and LDL and an increasingly significant in HDL level after treatment for the Type 2 diabetes which makes it a therapy useful therapeutic in the treatment and improvement of Type 2 diabetic patients.

\section{AUTHORS' CONTRIBUTIONS}

Gozif M. N. Omar has carried out experiments and analyzed results, Fadel Yousif Al-Arabi has analyzed results, Mansour A. H. Mehdi has analyzed and typing the manuscript, and Madhukar M. Fawade has guided and Supervise research.

\section{CONFLICTS OF INTEREST}

The authors declare that they have no conflicts of interest.

\section{REFERENCES}

1. American Diabetes Association. Diagnosis and classification of diabetes mellitus. Diabetes Care 2014;37 Suppl 1:S81-90.

2. Wild S, Roglic G, Green A, Sicree R, King H. Global prevalence of diabetes: Estimates for the year 2000 and projections for 2030. Diabetes Care 2004;27:1047-53

3. Kamala S, Mohammed A, Swathi K, Venkateswarlu K, Ram MR. Evaluation of pharmacoeconomic direct cost in diabetes patients. Asian J Pharm Clin Res 2017; 10:38-40.

4. Ting HH, Timimi FK, Boles KS, Creager SJ, Ganz P, Creager MA, et al. Vitamin $\mathrm{C}$ improves endothelium-dependent vasodilation in patients with non-insulin-dependent diabetes mellitus. J Clin Invest 
1996;97:22-8

5. Fadupin GT, Akpoghor AU, Okunade KA. A comparative study of serum ascorbic acid level in people with and without Type 2 diabetes in Ibadan, Nigeria. Afr J Med Med Sci 2007;36:335-9.

6. Afkhami-Ardekani M, Shojaoddiny-Ardekani A. Effect of Vitamin C on blood glucose, serum lipids and serum insulin in Type 2 diabetes patients. Indian J Med Res 2007;126:471-4.

7. Prajapat R, Bhattacharya I, Jakhalia A. Combined effect of Vitamin $\mathrm{C}$ and $\mathrm{E}$ dose on Type 2 diabetes patients. Adv Diabetes Metab 2017;5:21-5.

8. Matough FA, Budin SB, Hamid ZA, Alwahaibi N, Mohamed J. The role of oxidative stress and antioxidants in diabetic complications. Sultan Qaboos Univ Med J 2012;12:5-18

9. Bonnefont-Rousselot D, Bastard JP, Jaudon MC, Delattre J. Consequences of the diabetic status on the oxidant/antioxidant balance. Diabetes Metab 2000;26:163-76.

10. Omar G, Mehdi M, AL-Arabi F. Management of diabetes and its complications through the role of antioxidants: Review. Res Rev Int J Multidiscip 2018;9:519-26

11. Vinotha AT, Senthilkumaran P. Effect of enalapril and metformin on oxidative stress in newly diagnosed Type 2 diabetes mellitus and hypertensive patients. Asian J Pharm Clin Res 2015;9:69-72.

12. Srivatsan R, Das S, Gadde R, Manoj-Kumar K, Taduri S, Rao N, et al. Antioxidants and lipid peroxidation status in diabetic patients with and without complications. Arch Iran Med 2009;12:121-7.

13. Afkhami-Ardekani M, Vahidi AR, Borjian L, Borjian L. Effect of Vitamin C supplement on glycosylated hemoglobin in patients with Type 2 diabetes. J Shah Sad Univ 2003;10:15-8.

14. Ganesh ND, Chaudhari HV, Shrivastava M. Supplementation of Vitamin $\mathrm{C}$ reduces blood glucose and improves glycosylated hemoglobin in Type 2 diabetes mellitus: A randomized, double-blind study. Adv Pharmacol Sci 2011;2011:1-5

15. Forghani B, Goharian V, Kassayian N, Amini M. The effect of Vitamin $\mathrm{C}$ supplements on serum lipoprotein levels in NIDDM patients. J M C
Isfahan Univ 2001;2:95-100.

16. Eriksson J, Kohvakka A. Magnesium and ascorbic acid supplementation in diabetes mellitus. Ann Nutr Metab 1995;39:217-23.

17. Paolisso G, D'Amore A, Giugliano D, Ceriello A, Varricchio M, D'Onofrio F, et al. Pharmacologic doses of Vitamin E improve insulin action in healthy subjects and non-insulin-dependent diabetic patients. Am J Clin Nutr 1993;57:650-6.

18. Reaven PD, Herold DA, Barnett J, Edelman S. Effects of Vitamin E on susceptibility of low-density lipoprotein and low-density lipoprotein subfractions to oxidation and on protein glycation in NIDDM. Diabetes Care 1995;18:807-16.

19. Paolisso G, D'Amore A, Galzerano D, Balbi V, Giugliano D, Varricchio M, et al. Daily Vitamin E supplements improve metabolic control but not insulin secretion in elderly Type II diabetic patients. Diabetes Care 1993;16:1433-7.

20. Pazdro R, Burgess JR. The role of Vitamin E and oxidative stress in diabetes complications. Mech Ageing Dev 2010;131:276-86.

21. Iqbal S, Naseem I. Role of Vitamin A in Type 2 diabetes mellitus biology: Effects of intervention therapy in a deficient state. Nutrition 2015;31:901-7.

22. Hodis HN, Mack WJ, Dustin L, Mahrer PR, Azen SP, Detrano R, et al. High-dose B vitamin supplementation and progression of subclinical atherosclerosis: A randomized controlled trial. Stroke 2009;40:730-6.

23. Low-Income Americans and Racial and Ethnic Minorities Experience Disproportionately Higher Rates of Disease. U.S Medicine Report; 2009.

24. Cabrera-Valladares G, German MS, Matschinsky FM, Wang J, Fernandez-Mejia C. Effect of retinoic acid on glucokinase activity and gene expression and on insulin secretion in primary cultures of pancreatic islets. Endocrinology 1999;140:3091-6.

25. Ziouzenkova O, Orasanu G, Sharlach M, Akiyama TE, Berger JP, Viereck J, et al. Retinaldehyde represses adipogenesis and diet-induced obesity. Nat Med 2007;13:695-702 